\title{
PRIVATIZACIÓN DE LA PROVISIÓN EDUCATIVA EN CHILE: CARACTERIZACIÓN DE LOS PROVEEDORES EDUCATIVOS DE UNA COMUNA POPULAR URBANA*
}

\author{
JuAN GONZÁLEZ LóPEZ ${ }^{1}$ \\ Diego Parra Moreno ${ }^{2}$
}

\begin{abstract}
RESUMEN: La provisión privada ha estado presente en Chile desde los inicios del sistema educativo obligatorio a principios del siglo XX. Actualmente, el sistema está mayoritariamente controlado por los privados, aunque su financiamiento sea estatal. Esto se ha consolidado desde la Reforma Neoliberal desde el año 1982. La oferta particular subvencionada ha aumentado explosivamente desde el año 1995. ¿Quiénes son los sostenedores de estos establecimientos educacionales? El presente estudio avanza en la caracterización del sector a partir del análisis de las redes educativas, económicas, ideológicas y políticas que estos presentan, por medio de fuentes primarias y secundarias. Entre los principales resultados, se encuentran proveedores con fuertes vínculos religiosos, económicos, y secundariamente educativos y políticos, quienes se posicionan como un grupo de poder ante otros proveedores particulares subvencionados. Por lo tanto, este grupo es el que mejor se adapta a las regulaciones de la actual reforma educativa en el sistema obligatorio en Chile.
\end{abstract}

Palabras clave: Privatización de la educación. Proveedores educativos. Libertad de enseńanza. Escuelas particulares subvencionadas.

\section{Privatization OF EDUCATIONAL PROVISION IN CHILE: CHARACTERIZATION OF THE EDUCATIONAL PROVIDERS A POPULAR COMMUNITY}

ABSTRACT: The private sector has been present in Chile since the beginning of compulsory education in the XX century. Recently, the education system is largely controlled by the private sector, although it is state-funded. This situation has been reinforced since the 1982 Neoliberal Reform. The private subsidized education has dramatically increased since 1995 . Who are the providers (or stakeholders) of these educational

\footnotetext{
*Este artículo ha sido redactado en base al trabajo de investigación realizado en el equipo de investigación en privatización de la educación integrado por Rayen Alday, Rocío Herrera y Estibhaliz Backit de Centro ALERTA.

${ }^{1}$ Universidad de Chile, Departamento de Psicología y Departamento de Educación - Santiago, Chile.

E-mail: juangl@uchile.cl

${ }^{2}$ Universidad de Chile, Cooperativa de Trabajadores/as Centro ALERTA y Observatorio Chileno de

Políticas Educativas (OPECH) - Santiago, Chile. E-mail: dparram@ug.uchile.cl

DOI: 10.1590/ES0101-73302016146813
} 
institutions? Based on the analysis of the primary and secondary sources of Chilean educational, economic, ideological, and political networks, this study advances in the characterization of the private sector of education provision subsidized by the State. The research results indicate the strong presence of private providers with strong religious and economic connections. These education private providers constitute a power group in comparison to other state subsidized private providers. Therefore, this is the group that best satisfies the regulations of current educational reform in the mandatory educational system in Chile.

Keywords: Privatization of education. Education providers (stakeholders). Freedom of education. Subsidized private schools.

\section{LA PRIVATISATION DE L'OFFRE ÉDUCATIVE AU CHILE: CARACTÉRISATION DES PRESTATAIRES DE SERVICES ÉDUCATIFS D'UNE COMMUNALE POPULAIRE URBAINE}

RÉSUMÉ: La fourniture privée a été présente au Chile depuis le commencement du système d'enseignement obligatoire au début du XXe siècle. Actuellement, le système est largement contrôlé par des entreprises privées, même si le financement provient de l'État. Ce contrôle se consolidât depuis la réforme néolibérale dès 1982 . L'offre privée subventionnée a augmenté de façon explosive depuis 1995. Qui sont les détenteurs de ces établissements d'enseignement? Cette étude, à travers de l'utilisation de sources primaires et secondaires, approfondit la caractérisation du secteur à partir de l'analyse des réseaux éducatifs, économiques, idéologiques et politiques auxquels les détenteurs appartiennent. Parmi les principaux résultats, nous retrouvons des fournisseurs avec des liens religieux et économiques solides et deuxièmement avec des liens politiques et éducatifs. Ces fournisseurs se positionnent comme un groupe de pouvoir en relation à d'autres fournisseurs privés subventionnés. Par conséquent, ce groupe est celui qui s'adapte le mieux aux régulations de l'actuelle réforme éducatif dans le système obligatoire au Chile.

Mots-clés: Privatisation de l'éducation. Fournisseurs éducatifs. Liberté d'enseignement. Écoles privées subventionnées.

\section{Introducción}

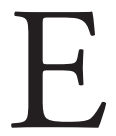

1 sistema educativo chileno, que es uno de los más privatizados del mundo, ha resultado ser un sistema efectivo, en tanto las reformas empleadas favorecieron a la subordinación total del significado y función educativa a las leyes del mercado, constituyéndose, desde su institucionalidad y finan- 
ciamiento, en una empresa capitalista que produce desigualdad y marginación. La liberalización de la provisión educativa ha tenido efectos adversos en los resultados del aprendizaje, en la pertinencia social de las escuelas y ha favorecido una marcada segmentación de la población escolar (ASSAÉL et al., 2011). Además, la desigualdad que la escuela privada reproduce se acentúa año tras año. En Chile, se ha consolidado un sistema de educación obligatoria universal de provisión educativa mixta, con un fuerte pilar privado subvencionado, el cual alcanzó, al 2014, una cobertura de un 54\% de la matrícula total del país, llegando en algunas comunas a más de un $80 \%$ del registro. La provisión educativa chilena contiene una paradoja, está financiada por el Estado, pero está controlada por privados.

\section{Reseña histórica de la provisión educativa mixta en Chile}

La provisión privada existe en Chile desde los inicios del período de la República (BRAHM; CARIOLA; SILVA, 1971). Desde su principio en manos de la Iglesia Católica, institución ligada al origen colonial de Chile, estrechamente vinculada a la elite chilena. En el siglo XIX, la Iglesia Católica mantenía, además de las escuelas para la elite, las llamadas escuelas confesionales, que recibían subvención estatal. Según Madero y Madero (2012), en 1864, existía un 33\% de escuelas públicas, mientras el $67 \%$ eran privadas católicas. Para los grupos conservadores y la Iglesia, la educación de los pobres pasó a tener importancia política hacia finales del siglo XIX, ante las primeras señales de la 'cuestión social' en Chile ${ }^{1}$. El historiador James Morris afirma que la elite asumía el deber de estar cerca del pueblo y ejercer el tradicional 'patronazgo'.

Debían conversar con ellos, escuchar quejas, ayudarlos en sus escuelas de manera tal que les hiciesen ver a los pobres lo absurdo de las utopías socialistas y le quitaran del cerebro la falsa idea de la igualdad mostrándole que hay un orden providencial, que es preciso respetar. (MORRIS, 1967, p. 114).

Tal principio doctrinario conservador fue el motor de la fundación y defensa de instituciones educativas y de beneficencia por parte de la elite rentista (MORRIS, 1967). La provisión mixta protagonizada fundamentalmente por la Iglesia Católica y los grupos conservadores de la elite, se fortaleció a través de la llamada libertad de enseñanza, un principio legal y filosófico que permitió resguardar la autonomía de las escuelas católicas.

Tras la Ley de Instrucción Primaria Obligatoria del año 1920, se fortaleció el rol estatal en la educación, que fuera el hito del triunfo de las facciones liberales de la elite, sobre los grupos conservadores, que sin embargo, como señalamos antes, mantuvieron el control sobre sus escuelas. La Constitución de la República de 1925, en su capítulo III, artículo 10, inciso 7, consagra la 'libertad de enseñan- 
za’ y, a su vez, da al Estado un rol preferente en la atención de la educación pública: es el consenso de la elite en el ámbito educativo.

El proyecto del desarrollo industrial nacional que se comenzó gestar en ese entonces, tuvo en la escuela estatal uno de sus pilares. Al año 1972, en pleno desarrollo del gobierno de la Unidad Popular, el estado chileno llegó a invertir el $7 \%$ del producto interno bruto (PIB) en educación. En el marco del proyecto socialista-desarrollista de este período, se fortaleció institucionalmente el sistema educativo, mejorando la cobertura educativa y apostando a la inclusión de cada vez más sectores sociales. Acerca del crecimiento del sistema educativo en esa época, el economista Manuel Riesco señala que:

Las matrículas totales se multiplicaron por más de cinco veces entre 1925 y 1974, mientras la población se duplicaba, o poco más. El aumento de las matrículas se acelera hasta alcanzar un crecimiento promedio anual de 5,4\% entre 1960 y 1974. La educación superior crece a un ritmo de $13 \%$ anual durante ese período, el que se acelera más aún a consecuencia del movimiento de reforma de 1968, luego del cual la matrícula universitaria se duplica en menos de cinco años. (2007, p. 61).

En la misma época, la cobertura de la educación privada alcanzó a menos del 20\% del total nacional (ELACQUA, 2011).

En el 1973, el gobierno de la Unidad Popular anunció una profunda reforma educativa, la "Escuela Nacional Unificada" (ENU), que consistía, en líneas gruesas, en fortalecer y articular el sistema estatal e incluir la participación de las comunidades locales y organizaciones sociales (RAMÍREZ; MUÑOZ; HURTADO, 1973). Esta reforma sepultaba definitivamente el tradicional poder que la Iglesia y las elites conservadoras tenían sobre el sistema educativo, por lo cual fue inmediatamente atacada y acusada de ser una 'propuesta marxista con objetivos totalitarios' (VERA, 2012), constituyéndose en un argumento más para que las elites promovieran y apoyaran el Golpe de Estado en Chile, ocurrido el 11 de septiembre de 1973, inaugurándose un gobierno dictatorial que duró 17 años.

Después del Golpe de Estado, se estancó el desarrollo del sistema estatal por varios ańos. En 1980, la Dictadura Militar, por medio del Decreto 3.166, traspasó los Liceos Técnicos del Estado, en ese entonces de excelencia, a gremios empresariales como: la Cámara Chilena de Comercio, la Cámara Chilena de la Construcción, la Sociedad Nacional de Agricultura y la Sociedad de Fomento Fabril, entre otros. En el 1982, comienza la municipalización, que bajo el argumento de la "descentralización", comienza a desmembrar el sistema estatal, traspasando la gestión de las escuelas a los sostenedores. Estos últimos municipios y/o entidades particulares que se definen como colaboradores de la labor educativa del Estado. Paralelamente, el dictador impone una nueva constitución, en la que se garantiza la libertad de enseñanza, por sobre 
el derecho a la educación, blindando así al nuevo sistema privado de educación. Se incentivó la apertura de escuelas privadas con fines de lucro, ya que según el economista diseñador de este sistema, Gerardo Jofré (1988), "el dolor pecuniario tiende a corregir vehementemente cualquier asomo de irresponsabilidad", agregando que "un sistema basado en establecimientos particulares con fines de lucro, tiende no sólo a financiarse, sino a obtener el máximo aprovechamiento de los recursos" (JOFRÉ, 1998, p. 209).

\section{Privatización de la provisión educativa en Chile}

Actualmente, menos del $37 \%$ de la matrícula total se encuentra en la educación estatal/municipal, el resto está en la educación privada subvencionada o la privada ${ }^{2}$. Mediante un juego entre oferta y demanda, y entre Estado y Mercado, se consolidó un sistema educativo mixto, con un fuerte pilar privado. El modelo neoliberal eliminó, al más bajo costo posible, todo vestigio del fuerte pilar estatal del modelo desarrollista ${ }^{3}$, mientras la privatización ha llegado a extremos, particularmente en comunas urbano-populares, donde hay una mayor concentración de población y pobreza. Según datos de la Fundación Sol (2011), una de las comunas más privatizadas del país, es Alto Hospicio, que sólo cuenta con un 3,4\% de los/as estudiantes en establecimientos municipales, el resto está en escuelas privadas. De acuerdo a las bases de datos del MINEDUC (2014) en la Región Metropolitana (RM), donde vive la mayor parte de la población, menos del $26 \%$ de la matrícula está en las escuelas del Estado. Las comunas urbano-populares son las más privatizadas, por ejemplo, en Puente Alto y Maipú sólo el 17,7\% de la matrícula es municipal. Otros casos son la comuna de La Pintana, con sólo un 17,9\% de la educación municipal (estatal) y La Cisterna con solamente un 11,5\% de la matrícula municipal. Por lo tanto, en estas comunas, más del $80 \%$ de la provisión educativa es privada, representando un desafío al Gobierno, que ha anunciado fortalecer la educación pública como un pilar fundamental del sistema educativo ${ }^{4}$.

\section{La investigación sobre los sostenedores del sistema particular subvencionado en Chile}

Frente al proceso descrito y la necesidad de fortalecer la educación pública, nos hacemos la pregunta ¿Quiénes son los privados que se hacen cargo de la provisión educativa subvencionada? La investigación en Chile ha sido escasa, a principios de los 2000 aparecieron los primeros indicios sobre este tema. Almonacid (2004), utilizando datos del Ministerio de Educación del año 2001, caracteriza a los sostenedores educacionales según el tipo de personalidad jurídica ${ }^{5}$ y el tipo de establecimientos que tienen. De esta forma, describió a los sostenedores particulares como: "los religiosos", principalmente adscritos a la religión católica, seguido de las iglesias evangélicas y otros credos; "los sociales", ligados a 
la beneficencia, como las fundaciones y corporaciones; y "los privados", desglosados en personas naturales y en sociedades anónimas, con fines de lucro 6 . Por otra parte, McEwan (2002) y posteriormente Elacqua (2006) proponen una clasificación de los sostenedores del sistema particular subvencionado en: particulares no religiosos con fines de lucro, que pueden ser tanto personas naturales como sociedades; particulares católicos, incluyendo congregaciones, parroquias y fundaciones religiosas; particulares protestantes, con la ramificación en iglesias metodistas, pentecostales y/o adventistas; y por último, particulares sin fines de lucro, como fundaciones, corporaciones, organizaciones no gubernamentales (ONGs) y universidades. Otra clasificación propuesta por Corvalán, Elacqua y Salazar (2009) plantea la existencia de sostenedores con fines de lucro, persona natural o jurídica, y sostenedores sin fines de lucro, como son las fundaciones o corporaciones, que pueden o no expresar una misión u orientación de tipo religiosa. La Tabla 1 presenta una síntesis de las clasificaciones señaladas.

La información respecto a las personas que ejercen directa o indirectamente la función de sostenedor es aún desconocida y no es posible saber con certeza cuántos establecimientos educativos sostienen indirectamente. El propio Almonacid (2008) advierte sobre la escasa información disponible para identificar "quiénes son los sostenedores, qué sociedades establecen, cuántos colegios administran a través de ellos y cuánto dinero reciben por concepto de subvenciones" (ALMONACID, 2008, p. 158). Quedan, entonces, muchas dudas respecto a estas clasificaciones, por ejemplo, el fin al lucro, es una dimensión ambigua y difícil

\section{Tabla 1}

Resumen de la categorización de los sostenedores particulares subvencionados.

\begin{tabular}{|c|c|c|c|}
\hline \multicolumn{2}{|c|}{$\begin{array}{l}\text { Sostenedores particulares } \\
\text { subvencionados }\end{array}$} & \multirow{2}{*}{$\begin{array}{c}\text { Lucro } \\
\begin{array}{c}\text { Sociedades anónimas, persona } \\
\text { natural y empresas }\end{array} \\
\text { Responsabilidad limitada }\end{array}$} & \multirow{2}{*}{$\begin{array}{c}\text { No lucro } \\
\begin{array}{c}\text { Congregaciones, fundaciones y } \\
\text { corporaciones }\end{array}\end{array}$} \\
\hline Relioinsos & Católicos & & \\
\hline 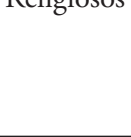 & Protestantes & $\begin{array}{c}\text { Sociedades anónimas, persona } \\
\text { natural y empresas } \\
\text { Responsabilidad limitada }\end{array}$ & $\begin{array}{l}\text { Parroquias, iglesias metodistas, } \\
\text { pentecostales y/o adventistas }\end{array}$ \\
\hline \multirow{2}{*}{$\begin{array}{l}\text { No } \\
\text { religiosos }\end{array}$} & Sociales & $\begin{array}{l}\text { Sociedades anónimas y } \\
\text { empresas } \\
\text { Responsabilidad limitada }\end{array}$ & $\begin{array}{l}\text { Fundaciones, corporaciones, } \\
\text { ONGs y universidades }\end{array}$ \\
\hline & $\begin{array}{l}\text { Privados/ } \\
\text { particulares }\end{array}$ & $\begin{array}{l}\text { Personas naturales, sociedades } \\
\text { anónimas y empresas } \\
\text { Responsabilidad limitada }\end{array}$ & Corporaciones y fundaciones \\
\hline
\end{tabular}

Fuente: Corvalán; Elacqua; Salazar (2009); Elacqua (2006); Almonacid (2004); Mcewan (2002). 
de indagar, ya que resulta de la definición jurídica asumida por el propio sostenedor. El caso de la educación superior en Chile es elocuente en este sentido. Todas las universidades privadas en el país son de instituciones que declaran no tener fines de lucro, sin embargo obtienen millonarias ganancias mediante triangulaciones y otros manejos financieros e inmobiliarios de los recursos ${ }^{7}$ (CHILE, 2008; 2012). Muchas universidades son ligadas a poderosos grupos empresariales (MÖNCKEBERG, 2005). Respecto la adscripción religiosa, esta categoría también es confusa, pues se sabe la adscripción formal a la iglesia en los establecimientos relacionados a las congregaciones, pero hay asociaciones de particulares católicos, o la simple adscripción a una determinada fe por parte del sostenedor. Hay incluso establecimientos municipales (públicos) que terminan operando como escuelas que promueven dicha fe o la evangélica, sin otras alternativas. Además, queda en una incógnita sus vínculos sociales, políticos o económicos, por ejemplo, si pertenecen a grupos de presión determinados, a ideológicos u otros. En este sentido, es necesario revisar estas clasificaciones, incorporando dimensiones que permitan hacer inteligible los grupos que controlan el sistema.

\section{La privatización: un fenómeno complejo}

Las investigaciones a nivel internacional sobre el fenómeno de la privatización aportan elementos para una clasificación más detallada, como lo descrito por Verger (2013), quién plantea la extensión de los llamados Public-Private Partnerships (PPP), o 'Alianzas Público-Privadas'. Las PPP son una relación contractual entre los sectores público y privado, en el que lo último se compromete a proveer un servicio, en este caso educativo, a un determinado precio y tiempo. Esta política, impulsada por el Banco Mundial, pretende, entre otros objetivos, desviar fondos destinados al gasto público, hacia los privados; fortalecer la alternativa privada en educación (considerada como de mejor calidad); generar más eficiencia en el manejo de "recursos" y debilitar los sindicatos docentes, entre otros beneficios, para la estrategia financiera transnacional (VERGER, 2013) .

Otro aporte es el que hacen Ball y Corbalán (2014), quienes ven en la privatización educativa una herramienta para constituir 'nuevas condiciones y tendencias para la gobernabilidad' en sociedades cada vez más complejas. Plantean que la presencia del sector privado se debe comprender en un nuevo contexto de políticas educativas, las cuales pasan de un sistema basado en el control burocrático a uno basado en contratos, de ventas de políticas educativas, atractivo para los gobiernos, como un medio para administrar resultados en vez de procesos. La contratación y descentralización de estos servicios introduce nuevos tipos de actores al ámbito de la función estatal, ampliando los espacios y modos de gobernar e introduciendo nuevos tipos de relaciones de poder. De este modo, se conformarían "redes y lazos duraderos que ofre- 
cen recursos clave de pericia, reputación y legitimación" (GRABHER, 2004, p. 104; apud BALL; CORBALÁN, 2013). Este nuevo tipo de gobernanza educativa se articularía desde "redes autoorganizadas e interorganizacionales caracterizadas por la interdependencia, intercambio de recursos, reglas del juego y autonomía significativa con respecto al Estado" (RHODES, 1997, p. 15; apud BALL; CORBALÁN, 2013). Así, los servicios públicos ensayan una composición de "alianzas estratégicas, acuerdos de trabajo conjuntos, redes, asociaciones y muchas otras formas de colaboración que traspasan las fronteras sectoriales y organizacionales" (WILLIAMS, 2002, p. 103; apud BALL; CORBALÁN, 2013) ${ }^{9}$.

En esta misma línea, la investigación sobre las 'tendencias de la privatización de la educación en Latinoamérica', de la Campaña Latinoamericana por el Derecho a la Educación (CLADE), se considera la privatización de la educación en nuestra región como un fenómeno multifacético, que no sólo se relaciona con el crecimiento de la provisión privada, y que

lo que está en juego no es solo el rol de la escuela privada o particular en el conjunto del sistema público de educación, sino más bien la deconstrucción de la lógica pública de los sistemas educativos, como resultado de la introducción de múltiples y diversas prácticas, valores, y agentes provenientes del mundo empresarial. (CLADE, 2015, p. 11).

En este complejo fenómeno, destacan en un nivel superior lo denominado como "la gobernanza corporativa", caracterizada por,

[...] la participación directa de representantes de corporaciones en la definición de la agenda de la educación pública, a través de mecanismos que no consultan los espacios de participación social puestos en marcha para la deliberación y la toma democrática de decisiones sobre la política pública. (CLADE, 2015, p. 21).

Mediante la constitución, esto lo hacen las redes de empresarios que buscan influir en la legislación y la provisión educativa, por medio de programas educativos financiados por empresas privadas o a través de su influencia directa de representantes de este sector en la toma de decisiones sobre la educación.

Desde la experiencia internacional, la privatización representa un fenómeno complejo que implica identificar redes, sus formas de gestiones económica y pedagógica, su relación con el Estado, sus vínculos con el sistema productivo actual y los grupos de poder político-ideológico. En esa línea, se hace necesario profundizar la indagación, incorporando también una mirada sobre estos elementos. 


\section{Otra forma de mirar los sostenedores de la educación obligatoria chilena}

Para avanzar en una mejor caracterización de los proveedores educativos en Chile, hemos decidido no basarnos únicamente en la personalidad jurídica que utilizan los proveedores o la finalidad que declaran, sino que además incorporar sus redes con el aparato productivo, así como sus relaciones con grupos de poder y sus prácticas educativas y políticas. Entre el período 2009 y 2010 (CORBALÁN; GONZÁLEZ; LIGÜEÑO, 2009; GONZÁLEZ et al., 2010), una primera caracterización fue hecha en la RM de Chile (región con más sostenedores en el país). Se revisaron las redes comerciales, políticas e ideológicas de una muestra aleatoria de personas (naturales o jurídicas) que tiene escuelas particulares subvencionadas, además revisamos las escuelas que estaban vinculadas (directa o indirectamente) a ellas, realizando así una primera caracterización del sector (GONZÁLEZ; LIGÜEÑO; PARRA, 2012b). Posteriormente, se incluyó una indagación longitudinal de los datos enfocados en una comuna (La Pintana, de la RM de Chile $)^{10}$. En esta ocasión, además de analizar las redes de cada sostenedor, número y tipo de escuelas, se adicionaron variables tales como la matrícula (Gráfico 1), los resultados educativos y la vulnerabilidad de la población escolar en 20 años (1995 y 2014).

\section{Gráfico 1}

Matrícula en educación pública y privada

de la comuna La Pintana, en Santiago de Chile, 1995-2014.

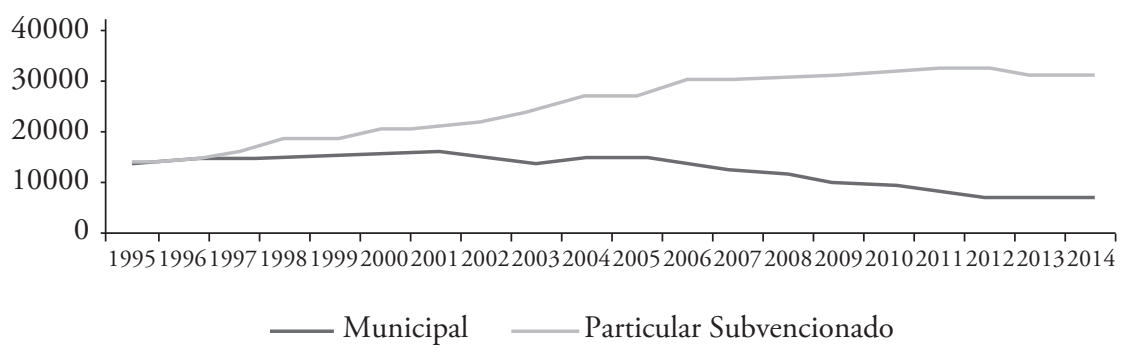

Fuente: Elaboración propia según base de datos (MINEDUC, 2014).

Por lo tanto, esta nueva información fue contrastada con la caracterización anterior, lo que nos permitió dar mayor densidad a las categorías ya trabajadas. Se condujo un análisis descriptivo de las fuentes primarias y secundarias de información del conjunto de sostenedores particulares subvencionados de la comuna de La Pintana. Tales fuentes corresponden a los registros del Ministerio de Educación, a los archivos del Ministerio de Justicia, registros del Servicios de Im- 
puestos Internos y del Diario Oficial de la República de Chile. Además, la concentración de matrículas y el número de establecimientos entre el año 1995 y 2014 fueron revisados, incorporándose variables asociadas a las escuelas como: existencia o ausencia del financiamiento compartido (FICOM) y puntaje del Índice de Vulnerabilidad Escolar (IVE) de cada establecimiento. Complementariamente, se aplicó un cuestionario por sostenedor (ficha) distinguiendo sus redes educativas, políticas, económicas e ideológicas, permitiendo su clasificación dentro de las categorías propuestas adelante. Estos son los resultados:

- Los grupos de poder: estrechamente ligados a los grupos de poder económico y político-ideológico, se vinculan en redes y logran incidir en la toma de decisiones sobre la educación pública. Participan o están vinculados con directorios de empresas controladoras de recursos naturales, industriales y financieros, además se vinculan a sectores conservadores de la Iglesia Católica. Por medio de las fundaciones y corporaciones sin fines de lucro, sostienen una o varias escuelas, en general de más de 1.000 alumnos, con edificios de buena infraestructura y equipamiento debido al financiamiento complementario que reciben vía donaciones del empresariado. Por ejemplo, en el bienio 2006-2007, de casi 30 millones de dólares que tuvo el fondo público de donaciones empresariales para la educación ${ }^{11}$, en la RM, el $90 \%$ se lo repartieron entre 55 escuelas a estos grupos, el resto fue a la educación del Estado, nada para otras escuelas privadas, de otro tipo de sostenedores. Así, año tras año se concentra este tipo de fondo en este sector. Tienen uno o varios colegios, que pueden estar en red, conectados entre ellos, mediante otras fundaciones o instituciones eclesiásticas y/o educativas. Constituyen redes entre ellos que coinciden en proyectos educativos, intercambio de recursos y patrocinadores ${ }^{12}$. Su conexión semeja a un rizoma ${ }^{13}$ (Diagrama 1), sin un centro común, que hipotetizamos se puede extender a través de algunas comunas y regiones, de todo Chile.

Este grupo interviene en la gestión de los establecimientos del Estado, asesorando municipios e implementando programas mediante las PPP. A esto se agrega la cogestión de Liceos Técnicos Públicos, en el que toman parte los gremios empresariales o las fundaciones ligadas a empresas ${ }^{14}$. Están muy relacionados a la toma de decisiones sobre políticas educativas, a través de la participación en mesas asesoras y cargos de influencia, son invitados a mesas técnicas convocadas por el Ministerio para la toma de decisiones educativas, han ocupado altos cargos ejecutivos y legislativos en el área de la educación pública o están vinculados a instancias de lobby en el marco de la Reforma Educativa. Este sector, que representa la gestión de escuelas de calidad en sectores de pobreza, es el grupo más fortalecido tras el debate educativo de estos últimos años. Por ejemplo, las escuelas de los grupos de poder en La Pintana controlan 


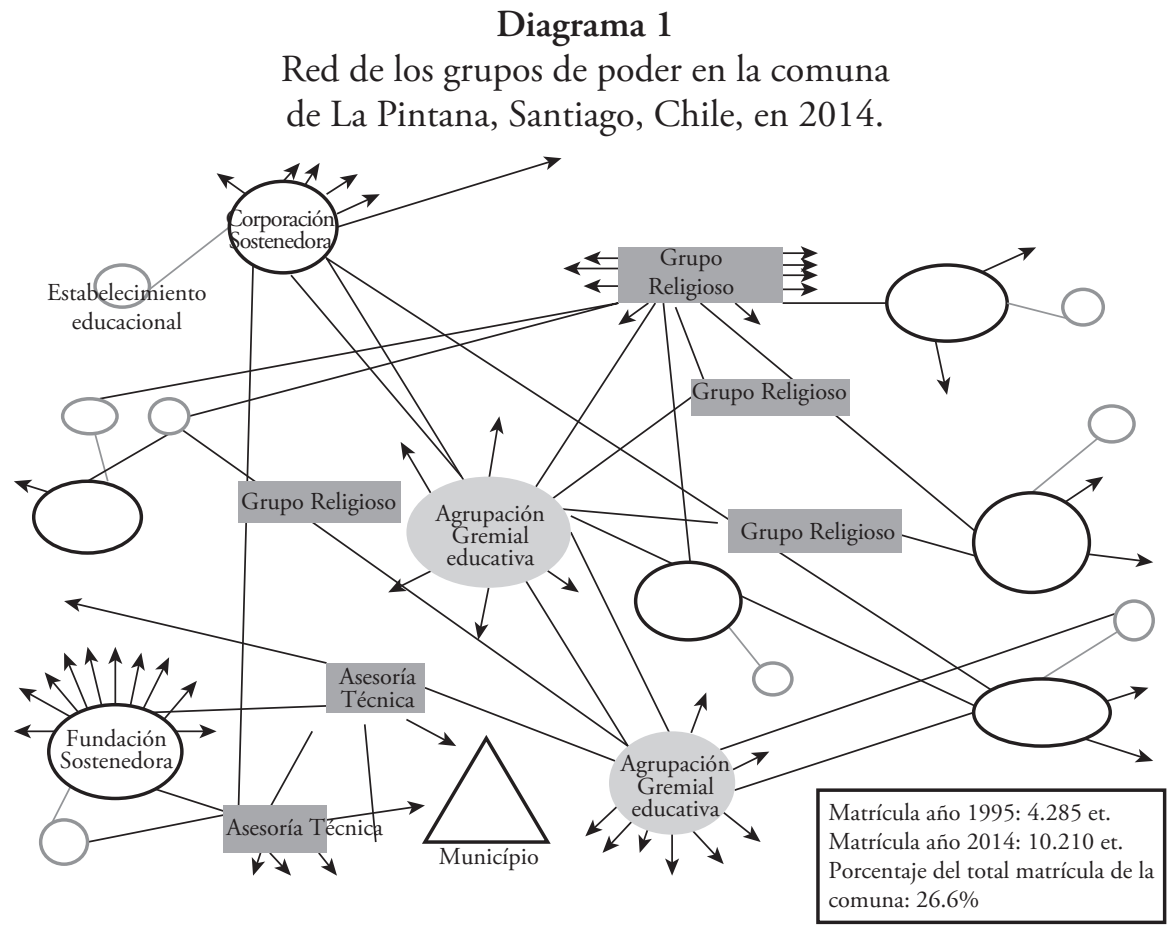

Las flechas indican relaciones con instituciones sostenedoras y/o educativas de otras comunas y/o regiones del Chile.

actualmente el 26,6\% de la matrícula comunal, lo que corresponde a la población escolar con menor IVE socioeconómico, si tomamos al grupo como conjunto ${ }^{15}$ (IVE de 71.89), mientras las escuelas municipales en la misma comuna atienden a la población con un IVE promedio de 81,87 puntos. Este grupo expandió su cobertura en la comuna en el período investigado. Se registró un aumento de la matrícula, que va de 4.285 estudiantes el 1995 para 10.210 el 2014, o sea, más que duplicó.

- Los inversionistas: aparecen vinculados directamente en más de una actividad con fines económicos, son miembros de directorios de empresas, sociedades anónimas y/o accionistas, resultando la escuela una actividad más entre otros rubros. No poseen vínculos claros con grupos económicos poderosos, ni políticos/ideológicos, aunque pueden tenerlos en forma secundaria. Algunos inversionistas se ligan a gremios como la Asociación Gremial Colegios Particulares de Chile (CONACEP) ${ }^{16}$. Han tenido la capacidad para invertir, desarrollando proyectos lucrativos y bien posicionados en el "mercado educativo". Tienen uno o más establecimientos grandes (más de 1.000 estudiantes), cobran FICOM ${ }^{17}$ y logran contar con mejor infraestructura e imagen corporativa que la 
competencia. Algunos de ellos son conocidos como los "mega sostenedores" 18 , ya que tienen varios establecimientos mediante sociedades anónimas o intermediarios. Son empresarios y/o comerciantes que ven en la educación un buen negocio, generan economías de escala y tienen capacidad para poner capital propio en principio, lo que les permiten obtener ganancias a mediano plazo ${ }^{19}$ operando algunos como verdaderos holdings educacionales. En la comuna La Pintana, este grupo, compuesto por siete entidades ${ }^{20}$, creció en matrícula y selectividad. Actualmente, controlan el 27,9\% de la matrícula comunal. En el ańo 1995, con dos entidades, atendían a 2.596 estudiantes. $\mathrm{Al}$ año 2014, alcanzan a 10.707 estudiantes con nueve establecimientos (Gráfico 2). Debido a la selectividad económica (cobran FICOM), atienden a un grupo de baja vulnerabilidad (70,59 puntos) en relación a los municipales. De los nueve colegios pertenecientes a los grandes comerciantes en La Pintana, ocho cobran una cuota mensual ${ }^{21}$, lo que excluyen a las familias más pobres.

\section{Gráfico 2}

Matrícula del grupo 'inversionistas' (1995-2014).

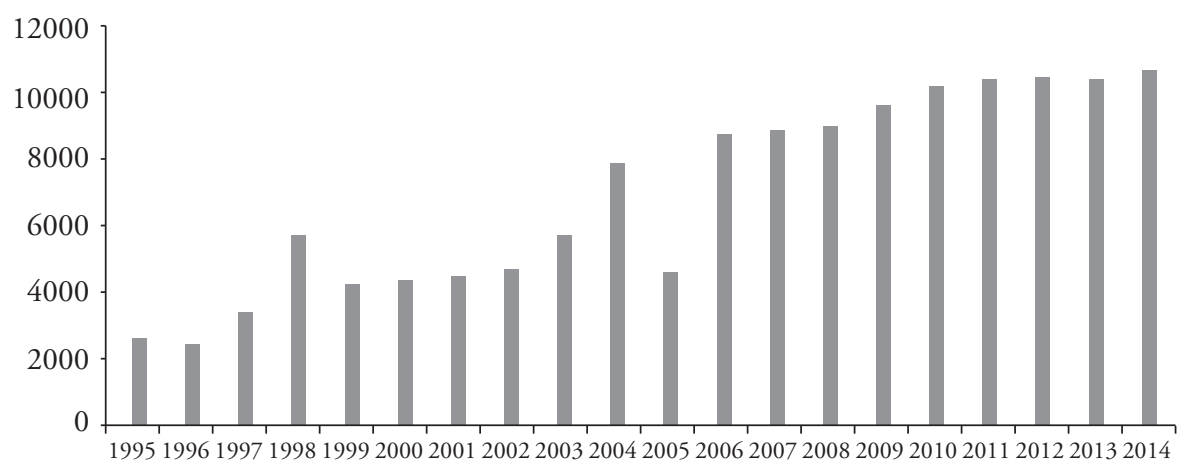

Fuente: Elaboración propia según base de datos (MINEDUC, 2014). El Ministerio de Educación no tenía información de matrículas entre los años 1999 y 2003 del sostenedor Luis Huneeus Madge. Sucede lo mismo el 2005 con la Sociedad Educacional Humboldt Limitada).

- Los comerciantes y asalariados: Estas dos categorías fueron descubiertas en el primer mapeo en la RM. Las trataremos en conjunto debido a los pocos datos disponibles que permiten distinguirlos entre ellos. $\mathrm{Al} \mathrm{mi-}$ rar su evolución como un grupo, son los que más han perdido en la competencia educativa, lo que se refleja en la matrícula de sus escuelas. Los comerciantes son los que aparecen vinculados a otras actividades económicas, como miembros de sociedades o relacionados directamente a la propiedad de negocios y otras empresas, sin mayores vínculos con grupos de poder. Sostienen escuelas (no más de dos), de menos de 1.000 estudiantes, por lo que el margen de ganancia que pueden obtener es 
menor, incapaces de montar economías de escala y terminan brindando una educación de baja calidad a los estudiantes más pobres. Cuesta diferenciarlos del segundo grupo denominado asalariados, debido a la dificultad de conocerse la vinculación que estos pueden tener con otras actividades económicas. Los asalariados tienen una escuela solamente (de menos de 1.000 estudiantes) y vivirían de su trabajo como sostenedores, e incluso, profesores del establecimiento. Por lo tanto, no tienen otros vínculos comerciales, ni de otro tipo. La escuela es su medio de subsistencia. Los datos asociados al comportamiento en 20 ańos de estos grupos en la comuna de La Pintana nos muestran que, salvo excepciones, han perdido matrícula. $\mathrm{Al}$ año 2005, con 15 establecimientos, estos grupos tenían matriculados a 7.400 estudiantes, al 2014 a través de 18 escuelas, bajaron a 6.012 estudiantes. Actualmente, estos grupos en Las Pintana se ven representados por 17 sostenedores con escuelas diversas, donde la más grande alcanza los 700 estudiantes y la más pequeña, 54 (una escuela de adultos). Constituyen el grupo en crisis frente al proceso de reforma, las normativas en discusión los afectan directamente, quedando al margen del debate educativo, de inversiones que los ayuden a mejorar y desvinculados de grupos de poderes económico, político e ideológico. Representan el 16\% de la matrícula comunal, un porcentaje similar a la municipal, y como en ella están los estudiantes más pobres.

- Los sostenedores de la Educación Especial: Se pudo identificar con claridad este grupo al revisar el caso de la comuna de La Pintana. Son distintos de los comerciantes y asalariados, ya descritos, por que reciben una subvención preferencial al trabajar con niños/as diagnosticados/as con necesidades educativas especiales (NEE). Aparecen como ofertantes, en su mayoría, tras las mejoras en el monto de la subvención a estudiantes con NEE descritas en el Decreto de Fuerza de Ley No2 (DFL 2) en el año 1998 (Gráfico 3) ${ }^{22}$. Tienen escuelas de modalidad de Educación Diferencial y/o Especial. Según la Ley General de la Educación (CHILE, 2009, Art. 23), la educación especial es aquella que entrega

\section{Gráfico 3}

Apertura de las escuelas especiales (1984-2014), en la comuna La Pintana.

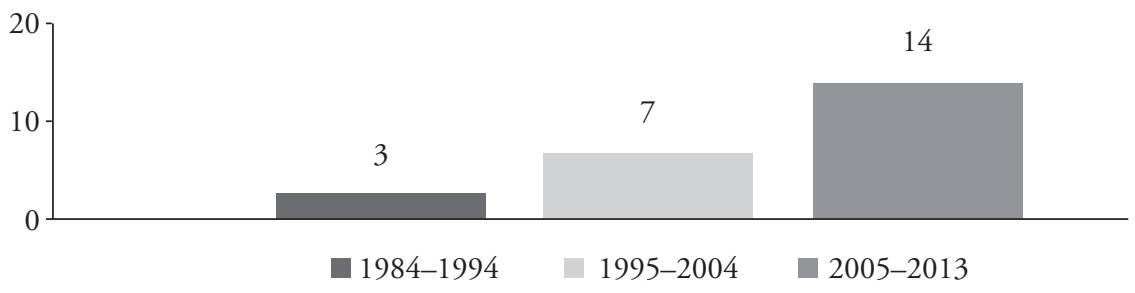

Fuente: Elaboración propia a partir de las bases de datos del Ministerio de Educación (MINEDUC, 2014). 
un conjunto de servicios, recursos humanos, técnicos, conocimientos especializados y ayudas para atender las NEE que puedan presentar algunos alumnos de manera temporal o permanente a lo largo de su escolaridad, como consecuencia de un déficit o una dificultad específica de aprendizaje.

En Chile, la oferta en esta área es casi toda privada. Actualmente, hay 1.817 escuelas especiales, sólo 153 son municipales $(8,4 \%), 1.661$ son particulares subvencionadas $(91,4 \%)$ y 3 particulares pagadas $(0,2 \%)$, como visto en el Gráfico 4.

\section{Gráfico 4}

Porcentaje de las escuelas especiales a nivel nacional según dependencia administrativa, en 2014.

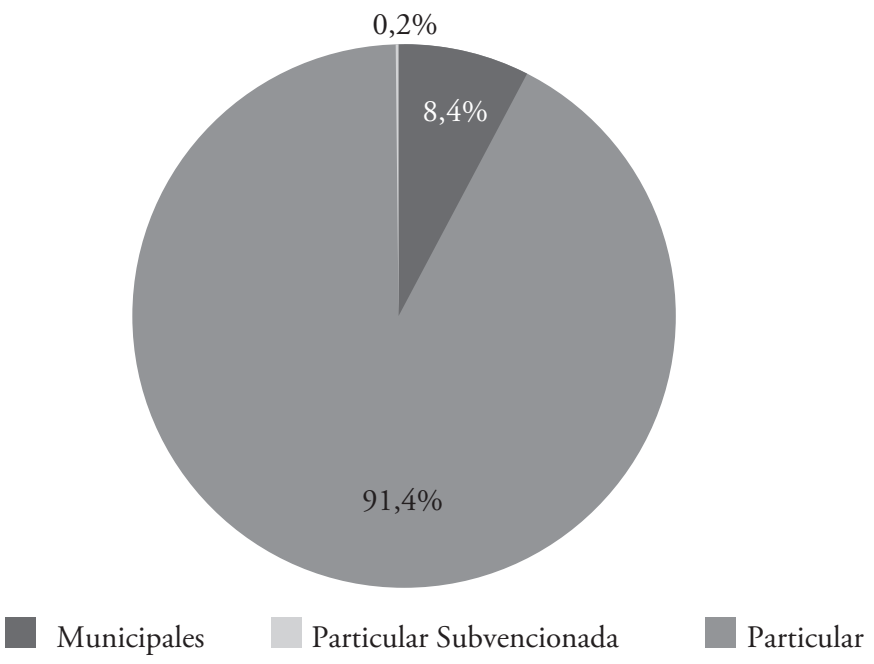

Fuente: Elaboración propia según base de datos (MINEDUC, 2014).

En el caso de la comuna de La Pintana, hay 24 sostenedores que imparten educación especial, representando un 39\% del total de sostenedores. Estos reúnen aproximadamente 6.000 estudiantes en conjunto, o sea un 19\% de la población estudiantil del sector particular subvencionado y un 15,6\% del total de las matrículas de la comuna. Han tenido un explosivo crecimiento en los últimos 20 años (Gráfico 5). En general, son escuelas pequeñas, la más grande tiene 500 estudiantes y la más pequeña, 25. Logran sustentarse gracias al monto de la subvención. La mayoría de los sostenedores posee sólo una institución y trabajan en ella. De los 25 sostenedores revisados, sólo cinco aparecen vinculados a otra escuela en otra comuna, siempre del mismo tipo y con similar cantidad de estudiantes. Es decir, se pueden considerar en la categoría de "comerciantes", descrita en el punto anterior, así mismo los que tienen solo una escuela y trabajan en ella serían asalariados. El tipo de escuela que sostiene, el monto de la subvención y su explosivo crecimiento, nos hace verlos como un grupo distinto. 
Otro tipo de sostenedores que pueden tener escuelas, desvinculados de los grupos de poderes político y económico vigentes, los cuales mediante figuras jurídicas diversas (personas naturales, sociedades anónimas, corporaciones, en general), pero no persigan salario, ni lucro, como organizaciones sociales, asociaciones de padres y/o maestros, cooperativas, otras iglesias, etc. son marginales, casi inexistentes en el sistema chileno.

\section{Discusión}

Según las categorías definidas en la comuna estudiada, hay una tendencia a la concentración de la matrícula en los "grupos de poder" y en "los inversionistas", quienes también educan a los/as niños/as con mejores condiciones socioeconómicas, o menor índice de vulnerabilidad. Creemos necesario avanzar en caracterizar mejor este grupo, ver si existen sub-grupos (poder religioso, empresarial y local, grandes y pequeños inversionistas), sus redes y su participación en la provisión educativa nacional. En el caso de La Pintana, es el sector que más se ha fortalecido y es probable que sea también así a nivel nacional. Los/as niños/as con más necesidades están en escuelas de los otros grupos (asalariados y/o comerciantes) o en la educación municipal. En el caso de esta comuna, el "grupo de comerciantes y asalariados" parece ser que iría a la baja, ya que no tienen redes económicas y/o político-ideológicas, significativas, ni vínculos entre ellos, quedan a merced de las condicionantes de mercado.

En este sentido, podría ser el grupo más afectado por las reformas en curso, sobre todo considerando que son los que están perdiendo matrícula y los más cuestionados en el debate educativo. En enero del 2015, se aprobó en el parlamento chileno una reforma que señala regular el lucro, remplazar el FICOM por un au-

\section{Gráfico 5}

Tendencia de las matrículas en escuelas especiales

(1995-2014), comuna de La Pintana.

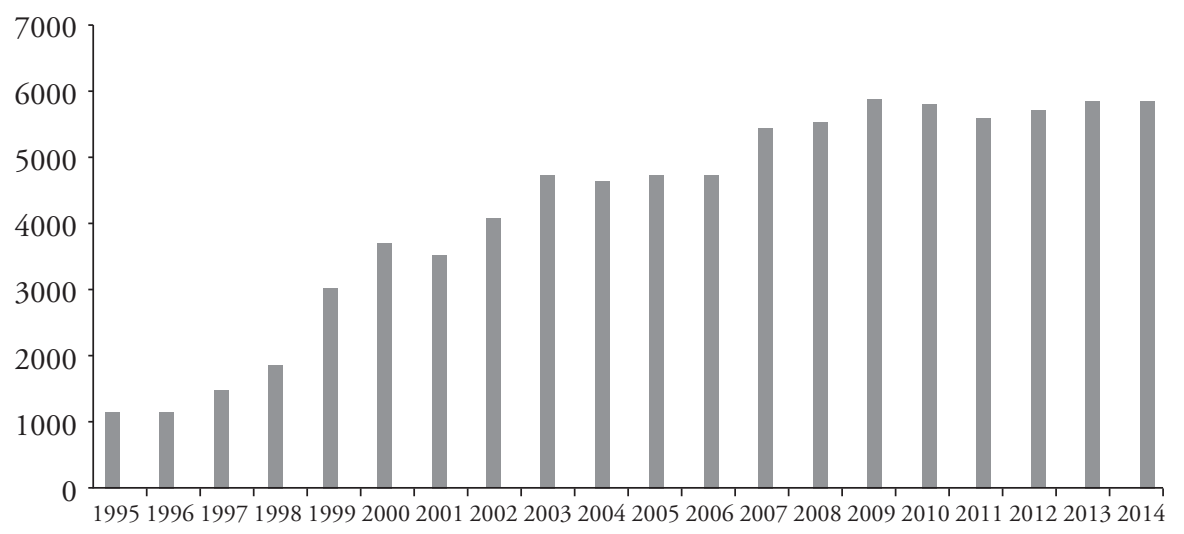

Fuente: Elaboración propia a partir de las bases de datos del Ministerio de Educación (MINEDUC, 2014). 
mento de la subvención estatal y terminar con la selección de estudiantes por parte de los sostenedores/establecimientos que reciben fondos del Estado, todo esto introduciendo mayor fiscalización del uso de los recursos y estableciendo mayores requerimientos institucionales a los sostenedores. ¿A qué grupos puede afectar en mayor medida este tipo de reforma? ¿Reducirán la privatización de la provisión educativa?

Respecto a la primera pregunta, creemos que lo más probable es que los "grupos de poder" y los "inversionistas" puedan adaptarse fácilmente en el nuevo escenario, tras personas jurídicas sin fines de lucro y una administración contable eficiente. Estos grupos tienen establecimientos grandes, incluso más de uno, hasta redes de escuelas, lo que les permitiría desarrollar economías de escalas, manteniendo márgenes de ganancia. Además, para algunos de estos, su interés es fundamentalmente ideológico (la iglesia y los grupos empresariales), o tienen otras inversiones que les permite no depender directamente de la subvención escolar, y así no verse afectado por los ajustes en su uso.

De implementarse la reforma ${ }^{23}$, el mayor problema lo podrían tener otros grupos, como los comerciantes, que con estos cambios podrían ser absorbidos por los más poderosos. Esto también es un fenómeno en el que se debe realizar un seguimiento y analizar más comunas. Mayor control en el uso de la subvención podría afectar el lucro que puedan extraer estos grupos - aunque según su tamaño y cantidad de escuelas, pueden cobrar por la función de "administración superior del establecimiento". Las restricciones en el FICOM y la selección no afectarán necesariamente a estos grupos, ya que en su mayoría no seleccionan y no cobran FICOM.

Otro caso es el de los "sostenedores que proveen educación especial". El crecimiento de este sector resulta un fenómeno evidente de privatización del sistema educativo en los últimos años, incentivado por la actual reglamentación (Decreto 170), lo que se refleja en la expansiva apertura de escuelas particulares de educación especial, desde que comienza regir esta normativa (1998), que entrega un incremento en la subvención a escuelas que reciben a niños/as diagnosticados/as con NEE. Cómo señalan los datos expuestos, el aumento del diagnóstico podría ser una consecuencia perversa de la privatización educativa y de la introducción de los mecanismos de mercado en el modelo educativo chileno. Hay que examinar este fenómeno y sus consecuencias en el etiquetamiento de niños/as, y revisar lo qué significa la alta presencia de este tipo de escuelas en comunas populares, como La Pintana.

Las reformas propuestas parecen no afectar la provisión educativa privada. Durante los últimos tres años, se han abierto 476 establecimientos particulares nuevos, 133 en 2015, pero entre el 2008 y el 2013 han desaparecido 420 escuelas municipales. Estamos lejos de revertir el proceso, si no se implementan reformas claras en dirección a fortalecer las escuelas del Estado, ninguno de los últimos anuncios parece ir en esa dirección. Podemos esperar, entonces, mayor privatización aún.

La desaparición del modelo educativo moderno, basado en un fuerte pilar estatal, implica el advenimiento de otro. ¿Cómo se configura este? Ya lo 
vimos sobre un fuerte pilar privado, sin embargo este es de naturaleza distinta al estatal. En él, participan distintos grupos, que representan diferentes (o ningún) proyectos educativos, que en el actual sistema de regulación, compiten entre ellos por la cantidad y el tipo de estudiantes.

En base a los resultados de la comuna de La Pintana, podemos suponer que el control del fuerte pilar privado de la educación chilena lo tienen los grupos de poder, entre ellos, con importante presencia, la Iglesia Católica, la elite del mundo empresarial y los grandes inversionistas. En perspectiva histórica, la privatización de la provisión educativa ha permitido una restauración conservadora en el control del sistema educativo. Tras la reforma neoliberal, la desarticulación del sistema estatal y la privatización de la provisión educativa, el sistema educativo expresa el estado actual de las relaciones de poder en la sociedad chilena capitalista, en la cual el poder económico, vinculado a los grupos religiosos conservadores, establece las condiciones de reproducción social ¿Qué rol le cabe al Estado en este contexto?

Seguimos en este sentido, la reflexión del Illich Ortiz, de quien nos permitimos hacer una extensa cita:

Si se trata de un orden democrático, es preciso que los poderes del espacio mercantil, que son en lo fundamental poderes privados, no invadan la esfera de lo público, ni cooptando el funcionamiento de las instituciones estatales en orden a servir sus intereses privados, ni cooptando la discusión en las arenas de la deliberación pública. En otras palabras, ni las empresas, ni sus fundaciones, ni su filantropía debe invadir el espacio ciudadano de la deliberación pública, en el cual la participación se debe llevar a cabo bajo razones de interés general. No es legítimo que las empresas y corporaciones utilicen su poder y sus recursos para hacer primar sus razones privadas (sus intereses y ambiciones particulares) en la esfera de lo público, ello implica una tergiversación profunda de la lógica que debe fundar un orden político democrático. (ORTIZ, 2013, p. 101).

Al Estado le corresponde, al menos, poner límites al poder privadomercantil. No basta regular el lucro, ni la calidad, como establece el discurso de la reforma educativa neoliberal. El sistema educativo público debe ser, entre otras cosas, democrático y plural, no puede estar controlado por el poder económico, ni religioso, ni tampoco orientado por el lucro. El Estado debe poner límites al mercado, no el mercado poner límites al Estado. Se debe tomar conciencia que la provisión educativa privada, como cualquier mercado que no es intervenido por el Estado, se concentrará en las manos de los más poderosos. El Estado debe retomar su rol igualador e intervenir en las desigualdades de origen, las cuales constituyen las bases sobre las que la libre competencia deviene en el control corporativo de los mercados y actualmente, en Chile, de la educación. 


\section{Notas}

1. La mayoría de los historiadores coincide que la cuestión social en Chile aparece entre 1880 y 1920, caracterizándose tanto por las malas condiciones que comienza vivir la clase obrera ante la crisis y el desarrollo del nuevo modelo productivo, como también por las distintas formas de organización que aparecen en el campo popular, para afrontar estas condiciones.

2. La matrícula particular pagada (sin subvención estatal) nunca ha superado el $9 \%$ de la matrícula total.

3. En 1980, la inversión estatal se había reducido al 4,6\% del PIB y, el ańo 1990, se redujo al 2,9\% en pleno proceso de expansión de la matrícula. El 2012, el Estado gastó apenas un 4,3\% de su PIB en educación, teniendo una cobertura total en las enseñanzas básica y media, que en Chile es obligatoria (OCDE, 2014).

4. El "Programa de Gobierno de Michelle Bachellet" (2013) señalaba como principio fundamental de la reforma comprometida: "El fortalecimiento de la Educación Pública como motor y sello del proceso de la Reforma Estructural. La Educación Pública debe fijar los estándares de calidad y tener presencia relevante en todo el territorio. Esta es la base y herramienta más potente sobre la que se construirá el proyecto educativo, la cohesión y la integración social" (BACHELLET, 2013, p. 17).

5. Según la reglamentación jurídica actual (Decreto Supremo 110), se define "personalidad jurídica" a un ente ficticio creado a partir de una asociación de individuos que persiguen un objetivo común, los cuales están dispuestos a cumplir obligaciones y ejercer derechos. Diferenciándose en las de derecho público (Estado, municipalidades) y las de privado con (sociedades civiles y comerciales) y sin fin de lucro (corporaciones y fundaciones).

6. ¿Qué es lucro en la educación? Se entiende que es la ganancia que se extrae de los recursos estatales y/o familiares destinados al funcionamiento de la escuela. Ganancia, beneficio económico o provecho que no resulta de un salario, en el caso que el sostenedor trabaje en la escuela, sino que resulta del privilegio que este tiene de manejar los suyos recursos.

7. ¿Cómo saber quién lucra? Actualmente en Chile se discute una reforma que prohíbe el lucro a los sostenedores, obligando a estos a adoptaren una personalidad jurídica sin fines de lucro, a invertir el dinero de las subvenciones en determinados ítems de gasto y fiscalizando la triangulación de recursos que estos hagan. Mecanismos costosos y que pueden ser fácilmente burlados por los grandes sostenedores (GONZÁLEZ; LIGÜEÑO; PARRA, 2012a).

8. En Chile, este tipo de alianzas público-privadas se expresa en diversos mecanismos como la cogestión de establecimientos educativos públicos, convenios de asesoría y dirección pedagógica, co-ejecución de políticas educativas, etc. Tales mecanismos son variados y van en aumento, pero tal tema excede el objetivo de esta investigación, aunque será abordada en otros trabajos.

9. En este sentido, es interesante para este estudio revisar las redes que tienen los sostenedores con organizaciones políticas y/o económicas, así como las redes entre ellos y con otros organismos educativos o de otro tipo.

10. Elegimos La Pintana debido a que es una comuna con uno de los peores índices de calidad de vida en Chile, con un alto índice de vulnerabilidad en la población escolar y por su alta matrícula en el sector particular subvencionado (82\%). El IVE es un indicador que va de 0 a 100, mientras el más alto 
el valor, mayor la vulnerabilidad. La matrícula municipal en la comuna en el año 2005 era de 15.394 estudiantes, al 2014, llega a 6.878 .

11. Mediante la ley 19.247 de donaciones con fines educacionales, el empresariado puede entregar dinero a estas escuelas, haciendo uso de beneficios tributarios.

12. Es el caso de la Fundación Educando Juntos, que reúne varias de las escuelas en el estudio y que se presenta como una red de intercambio pedagógico. En su directorio, se encuentran representantes del empresariado e incluso ex-ministras/os de la educación. Vinculado a más de 40 establecimientos, cuenta con el patrocinio del Banco Chile. Otro caso es la Fundación Arturo Irarrázaval Correa, que se enfoca en escuelas técnicas y también aparece vinculada a escuelas en el estudio, ella declara en su historia "la Fundación no pretendió abrir nuevas escuelas, sino que apoyar a las ya existentes. Sobre todo le interesaban las instituciones dependientes de corporaciones que asegurarán una orientación cristiana en la formación de sus alumnos". Actualmente, esta fundación patrocina más de 100 establecimientos, donde estudian 90.000 jóvenes. La Familia Irarrázaval es una familia colonial en Chile, vinculada a los grupos de poder económico actualmente, particularmente a sectores del capital financiero y la energía. Como estas, hay más instituciones que "enredadas entre sî", concentran el apoyo de grupos de poderes político, económico e ideológico (católicos conservadores). Véase <http://www.fundacionirarrazaval.cl/>.

13. Rizoma es una imagen o modelo descriptivo que se desprende de la botánica y designa un funcionamiento en red sin un centro común, ni jerarquía, que se extiende en determinado territorio o espacio.

14. En la Primera Región de Chile, en la comuna de Alto Hospicio, se encuentra el llamado "Liceo Minero", un Liceo Municipal Técnico Profesional de excelencia, el cual desde el año 2012 sostiene una alianza estratégica con la Fundación Educacional Collahuasi, la que participa activamente en la instalación de procesos en gestiones institucional y pedagógica a través de asesorías al liceo.

15. Nueve entidades sostenedoras en La Pintana conforman los llamados grupos de poder: Fundación Nocedal, Sociedad de Instrucción Primaria, Fundación Marcelino Champagnant, Fundación Didascolio, Fundación Padre Álvaro Lavín, Corporación Aprender, Corporación Arzobispado de Santiago, Fundación Belén Educa y la Corporación Educacional de la Construcción.

16. El Centro de Investigación Periodística (CIPER) publicó en junio del 2014 un reportaje denominado "Colegios subvencionados: así operan los siete grupos de 'megasostenedores' que lideran el negocio" aludiendo principalmente a este sector. Disponible en: <http://ciperchile.cl/2014/06/06/colegios-subvencionados-asi-operan-los-siete-grupos-de-megasostenedores-que-lideran-el-negocio/>.

17. FICOM: Desde el año 1993 se aprobó la posibilidad legal que los establecimientos particulares cobrasen una cuota a las familias que permitiese complementar la subvención que entrega el Estado. El FICOM se ha extendido a la mayoría de los colegios subvencionados e incluso a la educación municipal, segregando aún más a la población escolar.

18. Por ejemplo, aparece en nuestro estudio la Sociedad Educacional e Investigaciones Pedagógicas Galvarino Ltda., ligada al grupo Cifuentes Narváez, parte de los llamados “megasostenedores”. Este grupo que parte el año 1974 con Filomena Narváez, una de las fundadoras de CONACEP, hoy, en manos de su sus hijos, es un holding educacional, que tras diez sociedades distintas sostienen 19 establecimientos en otras comunas, además sostienen una Universidad Privada y son propietarios de 
distintas sociedades inmobiliarias, agropecuarias y comerciales. Otro ejemplo es Luis Huneeus que sostiene el Colegio Jorge Huneeus Zegers. Tiene sólo un establecimiento pero de más de 3.400 estudiantes, es el más grande en la comuna. El sostenedor es un político local y acaudalado empresario, militante del partido de derecha "Renovación Nacional" y actualmente es concejal de la comuna, vinculado a distintas actividades comerciales y empresariales.

19. "El negocio es tener colegios de más de 1.000 estudiantes, si no, no es negocio. Nosotros apostamos a obtener una ganancia anual entre un 15 a 20\%, no menos" (Entrevista a sostenedor ligado a este grupo y miembro de los CONACEP, año del 2009).

20. La Sociedad Educacional e Investigaciones Pedagógicas Galvarino Ltda., la Sociedad Educacional Bezal S.A., la Sociedad Educacional Humboldt Limitada, Luis Alejandro Huneeus Madge, Sonia Isabel Zapata Diez, la Sociedad Educacional Antumahuida Ltda. y la Sociedad San José De La Familia.

21. El único establecimiento de este grupo que no cobra FICOM es una de las escuelas de Sonia Zapata, la cual figura como escuela de lenguaje. Estas escuelas, como veremos más adelante, se sostienen en ventaja respecto a las regulares, debido a que reciben una subvención de más del doble de la subvención regular, pudiendo llegar incluso a más de 300 dólares por estudiante (la subvención regular llega a 125 dólares por estudiante aproximadamente).

22. El Decreto Fuerza de Ley 170 indica una subvención especial (que llega a más del doble de la subvención normal) a los niños/as que tengan algún diagnóstico de NEE.

23. La reforma educativa llamada "Ley de Inclusión" (2015) es un conjunto de modificaciones a otras leyes, para eliminar el lucro, el FICOM y la selección de estudiantes. Respecto al lucro, prohíbe ocupar la subvención en ítems que no sean educativos, pero establece un ítem "administración superior del establecimiento", que es el pago al sostenedor, según valor de mercado y tamaño y cantidad de establecimientos. Es decir, legítima el lucro. Respecto al FICOM, establece una subvención extra para los sostenedores que se acojan a la "gratuidad", propone un proceso "gradual" al que se acojan primero los colegios que cobran menos. Andrés Palma, encargado de la reforma educacional, por parte del Gobierno, señala que hay colegios que pueden estar 20 años más cobrando. Respecto a la selección de estudiantes, la situación es similar, la eliminación es gradual y se permitirán excepciones (emblemáticos y por proyecto educativo). Los reglamentos para operacionalizar estas medidas aún no se han hecho (febrero del 2016) y la ley debiese comenzar a regir en abril del 2016, lo que pone en duda su efectiva implementación.

\section{Referencias}

ALMONACID, C. Un cuasi mercado educacional: la escuela privada subvencionada en Chile. Revista de Educación, n. 333, p. 165-196, 2004.

La educación particular subvencionada como cooperadora del Estado. En: BELlEI, C.; CONTRERAS, D.; VALENZUELA, J. (Eds.) La agenda pendiente en educación. Profesores, administradores y recursos: propuestas para la nueva arquitectura de la educación chilena. Santiago de Chile: Programa de Investigación en Educación, Universidad de Chile, Unicef, 2008, p. 156-182. 
ASSAÈL, J. et al. La empresa educativa chilena. Educação e Sociedade, Campinas, v. 32, n. 115 , p. 305-322, 2011.

BACHELLET, M. Programa de Gobierno Michelle Bachellet 1914-1918. Oct. 2013. Disponible en: <http://michellebachelet.cl/programa/>. Acceso en: 21 nov. 2013.

BALL, S.; CORBALÁN, F. Un nuevo escenario para la política y el negocio educativos. Cuadernos de Pedagogía, n. 435, p. 1-5, 2013.

BRAHM, L.; CARIOLA, P.; SILVA, J. La educación particular en Chile: antecedentes y dilemas. Santiago de Chile: Centro de Investigación y Desarrollo de la Educación (CIDE), 1971.

CAMPAÑA LATINOAMERICANA POR EL DERECHO A LA EDUCACIÓN (CLADE). Mapeo sobre tendencias de la privatización de la educación en América Latina y el Caribe. São Paulo, 2015.

CHILE. Los desafios de la educación superior: Informe del Consejo Asesor Presidencial, 2008.

Informe de la comisión investigadora sobre el funcionamiento de la Educación Superior, 2012.

Ministerio de Educación. Ley General de Educación, 16 de Diciembre, 2009. Disponible en: $\leq$ http://www.leychile.cl>

- Ministerio de Justicia. Aprueba Reglamento sobre concesión de personalidad jurídica a corporaciones y fundaciones que indica. Decreto Supremo n. 110, 17 en. 1979.

CORBALÁN, F.; GONZÁLEZ, J.; LIGÜEÑO, S. Identificación de la propiedad y dinámica de la oferta educativa particular subvencionada de la región metropolitana. Revista MAD, n. 20, p. 110-127, 2009.

CORVALÁN, J.; ELACQUA, G.; SALAZAR, F. Elsectorparticular subvencionado en Chile. Tipologización y perspectivas frente a las nuevas regulaciones. Centro de Investigación y Desarrollo en Educación, Centro de Políticas Comparadas en Educación. Proyecto FONIDE 69. Santiago de Chile: Ministerio de Educación, 2009.

ELACQUA, G. Breve historia de las reformas educacionales en Chile (1813-presente): cobertura, condiciones, calidad y equidad. Ponencia presentada en el Seminario Internacional "Los Desafíos de un Desarrollo Integral”. Congreso Nacional, Valparaíso, 5 jul. 2011.

Enrollment practices in response to vouchers: evidence from Chile. National Center for the Study of Privatization in Education, 2006. Disponible en: <http://www.ncspe.org/ publications files/OP125.pdf>. Acceso en: 13 ago. 2007.

FUNDACIÓN SOL. El desalojo de la Educación Pública. Ideas para el Buen Vivir, n. 1, p. 1-13, 2011. Disponible en: <http://www.fundacionsol.cl/wp-content/uploads/2011/12/ Ideas-1-Institucionalidad-y-Desarrollo.pdf $>$. Acceso en: 29 nov. 2011.

GONZÁLEZ, J.; LIGÜEÑO, S.; PARRA, D. ¿Cómo poner fin al lucro en educación? En: Centro Alerta (ed.). 2011: aportes para interpretar una década de lucha por autoeducación. Santiago de Chile: Quimantú, 2012a, p. 113-122.

El proyecto educativo del complejo religioso empresarial y las políticas de la nueva derecha en educación. Revista Diatriba, n. 2, p. 34-43, 2012 b. 
GONZÁLEZ, J. et al. Gubernamentalidad y provisión educativa privada en Chile. Reflexiones a partir de la identificación de la propiedad y dinámica de la oferta educativa privada en la Región Metropolitana. En: OYARZÚN, K. (Comp.). Sujetos y actores sociales: reflexiones en el Chile de hoy. Santiago: Universidad de Chile; Vice-Rectoría de Investigación; Facultad de Ciencias Sociales, 2010, p. 67-97.

JOFRÉ, G. El sistema de subvenciones en educación: la experiencia chilena. Revista Centro Estudios Públicos, n. 32, p. 192-237, 1988.

MADERO, C.; MADERO, I. Elección escolar y selección estudiantil en el sistema escolar chileno. ¿Quién elige a quién?: el caso de la educación católica. Revista Mexicana de Investigación Educativa, v. 17, n. 55, p. 1267-1295, 2012.

McEWAN, P. Public subsidies for private schooling: a comparative analysis of Argentina and Chile. Journal of Comparative Policy Analysis: Research and Practice, n. 4, p. 189216, 2002.

MINEDUC. Bases de datos de 1995 al 2014. 2014. Disponible en: < http://centroestudios. mineduc.cl/index.php?t=96\&i=2\&cc $=2519 \& \mathrm{tm}=2>$. Acceso en: 17 mar. 2016.

MÖNCKEBERG, M. El negocio de las universidades en Chile. Santiago de Chile: Debate, 2005.

MORRIS, J. Las elites, los intelectuales y el consenso. Santiago de Chile: Pacífico, 1967.

ORGANIZACIÓN PARA LA COOPERACIÓN Y EL DESARROLLO ECONÓMICO (OCDE). Education at a Glance 2014: OECD Indicators. OECD Publishing, 2014.

ORTIZ, I. Potencia ciudadana, ámbito público y profundización de la democracia en participación ciudadana, políticas públicas y educación. En: LUNA, M. Participación ciudadana, políticas públicas y educación. En América Latina y Ecuador. OEI, 2013. Disponible en: <http://www.oei.es/PUBLICAS.pdf>. Acceso en: 17 mar. 2016.

RAMÍREZ, O.; MUÑOZ, P.; HURTADO, M. Documentos especiales: la crisis educacional. Santiago de Chile: Quimantú, 1973.

RIESCO, M. Se derrumba un mito. Chile reforma sus sistemas privatizados de educación y previsión. Centro de Estudios Nacionales de Desarrollo Alternativo (CENDA). Santiago de Chile: LOM Ediciones, 2007.

VERA, J. La ENU como representación de la lucha político-ideológica durante la unidad popular. Revista Divergencia, n. 1, p. 73-94, 2012.

VERGER, A. Nuevas tendencias de privatización educativa. Las alianzas público privadas en educación. 2013. Disponible en: <http://educacionparatodos.cl/wp/ wp-content/uploads/2013/03/Cuaderno-del-Foro-Equidad-y-Financiamiento-de-laEducaci\%C3\%B3n.pdf>. Acceso en: 7 ene. 2014.

Recibido en 05 de Marzo de 2015.

Aprobado en 06 de Febrero de 2016. 